\title{
En temps de crise majeure, la télécommunication peut-elle être le pivot de la restructuration urbaine?
}

\section{Gilles Willett}

\section{(2) OpenEdition}

Journals

Édition électronique

URL : http://journals.openedition.org/communicationorganisation/1729

DOI : 10.4000/communicationorganisation. 1729

ISSN : 1775-3546

Éditeur

Presses universitaires de Bordeaux

Édition imprimée

Date de publication : 1 novembre 1994

ISSN : 1168-5549

Référence électronique

Gilles Willett, «En temps de crise majeure, la télécommunication peut-elle être le pivot de la

restructuration urbaine? », Communication et organisation [En ligne], 6| 1994, mis en ligne le 26 mars 2012, consulté le 01 mai 2019. URL : http://journals.openedition.org/

communicationorganisation/1729; DOI : 10.4000/communicationorganisation.1729

Ce document a été généré automatiquement le 1 mai 2019.

(c) Presses universitaires de Bordeaux 


\title{
En temps de crise majeure, la télécommunication peut-elle être le pivot de la restructuration urbaine?
}

\author{
Gilles Willett
}

1 Le citadin tient la télécommunication et la diffusion d'information pour acquises, un peu comme l'air respiré ou les aliments achetés chaque semaine au supermarché. Chaque jour, les systèmes de télécommunication et de diffusion concourent à la survivance de la ville et de ses habitants. Ils recréent chaque seconde la certitude qui rend la vie urbaine agréable pour les uns et tolérable pour les autres.

2 Dans la solitude ou la plénitude urbaine, ces systèmes demeurent des points d'ancrage d'une large gamme d'activités. Ce sont aussi des lieux de références symboliques, des outils de travail et des moyens d'action pour chaque individu. Aujourd'hui, la ville est à la fois une place de marché, un centre de production et de consommation, un lieu de transaction et de transit, un espace de savoir et de culture, une scène où se jouent tous les drames humains, le site des traces de l'histoire et le milieu où s'expérimentent tous les types de sociabilité humaine.

3 La ville est donc un espace symbolique, interactionnel et, parfois, relationnel qui fait et défait les croyances, les valeurs, les perceptions, les attitudes et les comportements de ses habitants. C'est un milieu fragile de transformations incessantes qui amplifie ou neutralise, à la vitesse de l'électron, tous les types d'événements.

\section{La ville télé communicationnelle}

4 Les systèmes de télécommunication et de diffusion sont des composantes essentielles de l'espace urbain. Leur rôle est primordial dans la structuration des interactions entre tous les éléments du milieu urbain. Ils en sont même devenus une condition préalable à l'existence et à la réalisation d'un nombre croissant d'activités. 
5 C'est par l'intermédiaire de ces systèmes que les citoyennes et les citoyens participent à l'économie tant locale qu'internationale, et ont accès aux services de pointe et aux dernières innovations. Ces systèmes ont donc plus qu'une fonction de liaison et d'interaction entre des personnes. En fait, ils constituent un espace industriel commercial, financier et symbolique, éphémère mais toujours renouvelé, caractérisé par le flux d'information qui détermine le rythme du vécu urbain. En ce sens, ces systèmes constituent un espace symbolique stratégique de vie et de survie, car c'est par leur intermédiaire que son produit et transitent les connaissances et l'information.

C'est en ce sens que les systèmes de télécommunication et de diffusion sont devenus un espace codificateur de la réalité urbaine. Ils contribuent à structurer les significations de la vie collective et des comportements individuels. De plus, ils sont devenus l'espace invisible qui assure la cohérence des activités urbaines, une certaine cohésion sociale et un consensus volatil, constamment épiés et renouvelés par les sondages. Ce qui doit être fait pour assurer la continuité urbaine et celle des règles minimales de sociabilité dépend de plus en plus des systèmes de télécommunication et de diffusion.

7 Cet espace est aussi un lieu de mutation individuelle et sociale tant pour l'urbain de souche que pour les personnes qui s'offrent en adoption à la ville. Dans bien de cas, l'espace télé communicationnel urbain entraîne la solitude ou rend le recours au voisinage superflu. Il amplifi la désarticulation spatiale, temporelle et affective des relations interpersonnelles entre les citadins. Il standardise les comportements d'échange d'information entre les individus et entre tous les types d'entreprises et d'institutions. Ainsi, il conforte l'ordre établi et détermine un niveau de certitude qui varie selon les informations transmises.

8 L'environnement télé communicationnel urbain est simultanément local et mondial. Il crée un espace sans distance, un environnement sans lieu et un temps sans durée, tout en constituant un symbole et une métaphore de convergence et de globalité dans un espace réel éclaté. Cet espace autonome et artificiel est imperceptiblement devenu pouvoir d'organiser et pouvoir des organisations. Les différentes liaisons, que cet espace autorise, se fondent et se confondent avec les flux électromagnétiques ainsi qu'avec les intentions et les stratégies des utilisateurs.

9 Cet environnement, ses terminaux, ses systèmes et ses réseaux s'imposent de façon spontanée et instantanée à l'esprit. L'action de télé communiquer est devenue un mode de vie et de structuration des interactions individuelles et institutionnelles. Ainsi, la rencontre, face à face devient une obligation qui a ses lieux et ses rituels, afin de retrouver la durée indispensable au maintien de la relation.

10 C'est parce que les systèmes et les réseaux de télécommunication permettent de fabriquer de l'espace et du temps artificiels qu'ils génèrent de l'incertitude, de l'instabilité et des perturbations, tout en étant des moyens de structuration de la convergence et de l'interdépendance entre les composantes urbaines. Cet espace et ce temps artificiels déstabilisent le temps, l'espace et la durée biologique de l'être humain. Cette déstabilisation est d'ailleurs occultée par l'obsession de gagner du temps pour accroître la quantité d'activités réalisables, pour produire et gagner de l'espace et pour accroître notre pouvoir d'intervention. Cette déstabilisation est amplifiée par la contrainte de l'immédiateté qui pousse à l'accélération des processus sociaux et culturels.

11 L'espace télé communicationnel urbain n'est pas un lieu que l'individu s'approprie. C'est tout au plus un outil de formalisation des liaisons et des interactions qui nous obligent à 
des comportements pseudo-relationnels spécifiques, fonctionnels, utilitaires et extraordinairement limités, car contrôlés par la technologie. Cet espace, le citadin d'aujourd'hui en dépend. C'est son défi quotidien et en même temps sa source de certitude fragile. Sans cet espace, le citadin vivrait constamment dans l'angoisse et ne pourrait pas maintenir l'illusion qu'il partage une même réalité avec les autres, peu importe la signification de cette réalité.

12 En milieu urbain, le temps et l'espace télé communicationnels fabriqués sont devenus une manière de raisonner sur la construction et l'évolution du monde. Regarder une ville à partir d'un avion ou d'une photo prise par satellite fait penser à un microprocesseur ou à un circuit imprimé. La ville télé communicationnelle pousse à l'algorithmie des raisonnements et des comportements humains.

\section{Lorsque la ville s'éveille}

13 Quand les radioréveils se déclenchent à l'unisson chaque matin, les nouvelles transmises à propos des événements, de la météo, de la circulation automobile et de la bourse déterminent déjà le climat de sociabilité dans plusieurs sphères d'activités. Au cours des ablutions matinales et du petit-déjeuner, des émotions sont brutalement réveillées, des attitudes sont, plus ou moins consciemment, structurées ou renforcées, des croyances et des valeurs sont mises en cause, des simulations de comportements sont élaborées et analysées, la planification de la journée est confirmée ou doit être revue. La télévision vient tempérer l'agitation matinale des jeunes ou montrer ce qui a été entendu à la radio.

(a) coup de teléphone de la journée apporte son lot de bonnes ou de mauvaises nouvelles au citadin. Il peut aussi servir à faire venir un taxi pour se rendre à l'aéroport ou au travail. En hiver, le système de télé démarrage prépare la voiture à recevoir son conducteur et ses passagers. Le système d'alarme contre le vol, le feu ou le bris d'une canalisation d'eau, relié à la centrale de surveillance, est activé. Le répondeur automatique est remis à zéro.

15 Au cours du trajet entre la résidence et les lieux de travail des conjoints, avec un détour par le lieu de garde ou d'étude des enfants, la radio débite son flux de nouvelles matinales. La circulation automobile et la météo sont pires ou mieux que ce qui est annoncé à la radio. Puis, c'est le bouchon causé par une panne de l'ordinateur qui règle le rythme de la circulation des véhicules et des piétons. Des policiers à chaque carrefour, reliés entre eux par un système émetteur-récepteur portatif, tentent de reproduire l'algorithme de la circulation véhiculaire à l'heure de pointe. Des conducteurs de voiture commencent alors leur journée de travail au moyen de leur téléphone cellulaire.

Les balises du système de transport en commun enregistrent des retards. Le répartiteur, suite aux informations que lui fournit son écran cathodique, s'affaire à déterminer de nouvelles routes pour certains véhicules. Puis, par l'intermédiaire d'un système de radiocommunication, il donne ses ordres aux conducteurs concernés afin d'essayer de maintenir l'image d'efficacité du transport en commun. Chaque chauffeur de taxi écoute les ordres du répartiteur transmis par le système de radio répartition et se demande si tel ou tel confrère vient d'obtenir la course la plus payante de la journée.

17 Un grave accident de la circulation impliquant plusieurs voitures et camions bloque une voie rapide. En quelques secondes, l'accident est signalé au service central des urgences, le 911, par les personnes qui disposent d'un téléphone cellulaire dans leur voiture ainsi 
que par les cébistes sur le canal 9. Les hélicoptères de la police et des stations de radio et de télévision ont déjà repéré l'endroit de l'impact. Peu de temps après les policiers et les ambulanciers arrivent sur les lieux. À l'hôpital le plus proche, les urgent logues entendent le blip blip de leur système de radiomessagerie. Ils se précipitent vers les appareils qui leur permettent d'entendre le rapport des ambulanciers. Pendant les échanges avec ces derniers, ils surveillent des appareils de télémétrie qui, depuis le système de radiocommunication de l'ambulance, indiquent déjà l'état des fonctions vitales du blessé transporté vers l'hôpital. En même temps, différentes interventions sont envisagées par les médecins. Arrivée à l'hôpital, la victime est branchée sur différents appareils qui surveillent et maintiennent ses fonctions vitales.

18 À l'aéroport, un système automatique de diffusion débite le numéro de chaque vol, sa destination et le lieu d'embarquement. Successivement, des pilotes reçoivent l'autorisation de décoller de la part des aiguilleurs du ciel juchés dans leur tour. Les membres de la sécurité se transmettent des informations par l'intermédiaire d'un système de radiocommunication dédié. Les passagers et leurs bagages à main sont passés au détecteur et aux rayons $\mathrm{X}$. Les imprimantes des ordinateurs de chaque compagnie d'aviation crépitent pour imprimer les cartes d'embarquement. Les points de ravitaillement des avions reçoivent leurs commandes sur écran cathodique. L'ordinateur, auquel sont reliées les caisses-terminaux des boutiques hors-taxes, enregistre chaque transaction et l'heure à laquelle elle s'effectue, et fait l'inventaire en temps réel. Un système de téléinformatique situé à l'autre bout du monde permet de contrôler en temps réel la carte de débit ou de crédit de chaque client.

19 À l'autre bout de la ville, une déflagration est entendue. L'explosion s'est produite dans une usine de produits toxiques. Peu de temps après leur arrivée, les policiers et les pompiers contactent par radiocommunication les ambulanciers et la sécurité civile. La cellule de crise de la mairie est activée. Des experts décident de lancer le plan d'évacuation d'urgence des résidents d'un quartier, car les responsables de la météo confirment que les vents dirigent les fumées toxiques vers ce quartier. Tous les intervenants, en pareil cas, sont inter reliés par des systèmes de télécommunication et chacun donne ses ordres, selon l'évolution de la situation décrite par les personnes sur les lieux, dont les journalistes. Les nouvelles diffusées par les médias provoquent alors la saturation du réseau téléphonique, car la parenté et les amis des sinistrés sont en quête de certitude. Les standardistes des hôpitaux et des différents systèmes d'intervention sont débordées.

20 Dans les gratte-ciel du centre ville, dans les usines, dans les commerces, dans les banques, dans les centres de recherches, les ordinateurs reçoivent et traitent l'information, les écrans cathodiques affichent les résultats du labeur algorithme des ordinateurs, les imprimantes déroulent des milliers de signes, les téléphones ne dérougissent pas, les systèmes de surveillance et de contrôle sont sur le qui-vive, les transactions amorcées à mille lieux de là, avant l'aube, continuent. Chaque personne joue son rôle dont le scénario dépend, en grande partie, de technologies électroniques qui déterminent les attitudes, les comportements, les attentes et l'humeur de chacune.

21 La ville, ce lieu aux comportements algorithmes, vie, survie, éclate, se recompose, évolue, s'agrandit, se suffit à elle -même, malgré les péripéties habituelles. L'urbain, une fois de plus, relève son défi quotidien d'exister, de produire, de consommer et de se détendre avec l'aide de la télécommunication et des ordinateurs. Pour la ville télé communicationnelle et ses citadins, il n'y a plus d'hier, de maintenant ou de demain. Il 
n'y a que l'instantanéité de l'électron et un environnement télé communicationnel mondial, artificiel, invisible, éphémère et constamment renouvelé.

\section{Lorsque la ville s'effondre} sirènes, dans les circonstances. certaines rues. savent pas à qui ils s'adressent.

Nous sommes le 17 octobre 1989 au nord de la Californie. Il est 17 heures 03, les répartiteurs du 911 exécutent leur travail routinier pour venir en aide aux personnes qui ont besoin de secours. Une minute plus tard, tout chavire, c'est le tremblement de terre de Loma Prieta. La ville télé communicationnelle n'existe plus.

Selon ce que rapporte Danita L. Osborne (Osborne, 1990), Public Safety Communications Instructor for the City of San José Communications Management Division, pendant une période de cinq à trente minutes après le tremblement de terre, tous les moyens sophistiqués de télécommunication sont inopérants. Les installations électriques municipales ont été détruites et les sources autonomes d'alimentation en électricité sont insuffisantes.

Le 911 du service central des urgences n'est plus opérationnel parce qu'il y a surcharge du nombre d'appels. Les systèmes d'alarme sonore interreliés au 911, de même que les autres systèmes de surveillance et de contrôle du lieu de travail des répartiteurs, sont débranchés. Ces derniers ne peuvent plus supporter le tintamarre des sonneries et des

5 Le système informatisé de répartition des secours est en panne. Il faut donc utiliser la répartition manuelle, ce à quoi les répartiteurs ne sont pas habitués. La réserve de cartes d'enregistrement des appels téléphoniques est insuffisante. De plus, il n'y a pas de cartes routières de la ville et les nouveaux répartiteurs ne savent même pas où se trouvent

Le système d'enregistrement des conversations téléphoniques est en panne, lui aussi, et ne peut être remis en marche. Les canaux dédiés de télécommunication pour joindre l'Office of Emergency Services (OES) ne sont plus opérationnels. Pour établir la liaison avec l'OES, il faut utiliser un vieux standard téléphonique dont les répartiteurs ne connaissent pas le fonctionnement. Ils n'ont pas été entraînés à cet effet. Dans certaines municipalités touchées par le tremblement de terre, de 3 à 4 jours sont nécessaires avant de pouvoir établir un lien télécommunicationnel avec l'OES.

7 Lorsque le réseau téléphonique redevient opérationnel, il est vite saturé d'appels. Dès lors, les portions du réseau qui fonctionnent sont chargées de friture, et les répartiteurs ont beaucoup de difficulté à comprendre leurs interlocuteurs. De plus, bien que les citoyens réussissent à joindre le 911, il est impossible d'établir une liaison avec les services compétents pour qu'ils puissent intervenir efficacement.

À San Jose, le centre de télécommunication est situé au quatrième étage d'un édifice qu'il faut évacuer. Le système d'air conditionné ne fonctionne plus et l'édifice est endommagé par le tremblement de terre. Les répartiteurs du 911 ne savent pas où sont situés les postes de commandement des services d'urgence. Lorsque 30 minutes après l'événement, ces postes redeviennent opérationnels, leurs opérateurs utilisent un acronyme pour s'identifier. Or, les répartiteurs ne connaissent pas ces acronymes et, par le fait même, ne

29 Les répartiteurs travaillent 12 heures consécutives et, de plus, ils sont responsables des relations avec les médias. Ils ne reçoivent pas d'information ou de mise à jour sur l'état 
des routes et les conditions de la circulation. Ils ne savent pas que certains ponts sont fermés. Ils ressentent un profond sentiment de frustration lorsqu'ils constatent que les médias donnent de l'information qu'eux n'ont pas et qui leur est indispensable pour bien faire leur travail.

Des numéros de téléphone importants comme ceux de la Croix Rouge, de l'office of Emergency Services, du Pacific Gas and Light, des inspecteurs d'édifices et des intervenants institutionnels en cas d'urgence sont périmés ou toujours engagés. Plusieurs personnes font, en même temps, l'état de la situation ou vérifient des informations, sans coordonner leurs efforts. D'où une grande confusion et un problème de validité des informations disponibles, de même que la difficulté de séparer les faits de la fiction.

31 Les répartiteurs sont entraînés à contrôler leurs émotions et leurs réactions ainsi qu'à faire abstraction de leurs problèmes personnels, afin d'apporter des secours adéquats. Malgré cela, certains deviennent hystériques, suite au tremblement de terre ou au stress provoqué par la situation qui prévaut au 911, et quittent le travail.

Ceux qui sont en poste ne reçoivent aucune image de télévision. Ils doivent donc se fier aux rapports oraux de plusieurs personnes et se faire une image personnelle de l'état de la situation. Certains considèrent que la situation en est une de destruction massive, ce qui les rend inaptes à aider le public et à sécuriser les personnes qui téléphonent au 911. D'autres estiment que c'est un tremblement de terre mineur et ne comprennent pas l'hystérie de leurs collègues et encore moins celle des gens qui parviennent à les rejoindre par téléphone.

33 Selon les règles établies, il doit y avoir, dans les 48 heures après l'événement, un support psychologique pour aider les répartiteurs à gérer leur stress. Il leur faut attendre trois jours. Lors de ces sessions, les membres de l'équipe de support psychologique identifient les problèmes mais n'apportent aucune solution. De plus, les répartiteurs ont le sentiment de ne pas être écoutés.

Les faits dont nous venons de faire état mènent au constat que, malgré la formation du personnel, malgré les plans d'urgence, malgré les systèmes de télécommunication sophistiqués, lorsque tout chavire, ce sont à la fois les structures mentales, symboliques et organisationnelles qui s'effondrent. Chaque individu retrouve alors sa fragilité dans un monde urbain de progrès qui, pendant une certaine période, a pour signification le chaos, l'abandon, l'incertitude, l'angoisse, le désordre. L'obsession organisationnelle millénaire de l'être humain fait place à l'obsession de la survie. Chaque personne redécouvre alors, pour un temps, l'importance de la relation humaine et de la compassion.

Mais ce n'est pas tout. Comme le fait remarquer le sergent Tom Watson (Watson 1990) du Santa Cruz, California, Police Department, la capacité des services d'urgence de fonctionner efficacement, lors d'un désastre de cette envergure, détermine la différence entre la vie et la mort. La possibilité d'établir et de maintenir une liaison, entre ceux qui ont besoin d'aide et ceux qui sont capables de la fournir, détermine l'efficacité du plan d'urgence.

Selon ce que rapporte le sergent Watson, tout s'est relativement bien passé après les premières minutes de panique qui suivirent l'événement. La bonne gestion du personnel et des tâches a évité l'épuisement des individus. Les liaisons électromagnétiques inopérantes ont été remplacées par des coursiers. Ces derniers avaient pour tâche de retracer les personnes présumées disparues, de livrer en main propre des messages ou encore de rechercher de l'information. 

lévénement. Selon John S. Powell (Powell, 1990), Emergency Preparedness Coordinator, University of California, Berkely, la couverture des médias a laissé croire que le tremblement de terre de Loma Prieta avait été aussi destructeur que ceux de Mexico ou d'Arménie. La plupart des médias n'étaient pas préparés pour un tel événement. Suite au tremblement de terre, la plupart des stations de radio et de télévision ne diffusaient plus. Elles n'étaient pas dotées de systèmes autonomes d'alimentation électrique. Elles étaient situées dans des édifices à risque, les équipements n'étaient pas harnachés et leurs antennes étaient situées dans des lieux ne pouvant supporter les secousses séismiques. Lorsque les stations de radio et de télévision reprirent l'antenne, les gens se sont sentis les otages des médias, car les systèmes de télécommunication ne fonctionnaient plus.

43 Selon Powell, la perception à long terme que les gens vont conserver de cet événement sera celle transmise par les premiers reportages des journalistes, malgré les nombreuses corrections et les faits rapportés par la suite. Les médias ont fait de cet événement le 
tremblement de terre du siècle. Même si la connaissance des faits s'est précisée avec le temps, l'information adéquate n'a pas été transmise par les médias.

Pour Powell, il importe donc que les services d'urgence définissent et intègrent dans leurs plans une fonction d'information publique d'urgence. Un des nombreux aspects de cette fonction, c'est le contrôle de la rumeur. Avant l'événement, des communiqués de presse devraient être préparés, selon certains standards, en y laissant les espaces appropriés pour y inclure, lorsque l'événement se produit, les informations pertinentes et vérifiées.

Les responsables de la fonction information publique d'urgence devraient être des personnes qui travaillent déjà de manière continue avec la presse. Les journalistes devraient être familiers avec les procédures de l'information publique d'urgence et participer à des exercices de simulation. Quant aux élus et aux responsables officiels, ils devraient être avisés sur ce qui peut être diffusé et sur ce qui ne peut l'être.

\section{Lorsque les décideurs s'embrouillent}

Transportons-nous maintenant au Québec, en août 1988, lors de l'incendie d'un entrepôt de BPC à Saint-Basile-Le-Grand, une petite municipalité située à environ 30 kilomètres de Montréal. Lors de cet événement, l'organisation fonctionnelle et hiérarchique des interventions est, selon Leroux (Leroux, 1989), spontanée plutôt que planifiée, sauf dans le cas de la mobilisation des ressources et de l'organisation fonctionnelle des corps de sécurité publique, tout au moins au début de l'incendie.

L'alerte dans le réseau de la santé et des services sociaux est déclenchée par hasard et sans procédure logique, retardant ainsi la mobilisation des ressources. Peu de personnes savent et comprennent comment et pourquoi certaines structures d'intervention sont mises en place, comment et pourquoi certaines décisions sont prises.

La coordination est assurée par des comités dont les rôles et les tâches sont définis selon l'évolution de la situation et la fréquence, plutôt épisodique, des réunions. La communication entre les membres des différents comités est assumée par des agents de liaison. Gouvernements, ministères et organismes travaillent en parallèle.

Plusieurs intervenants n'ont aucune formation préalable. Plusieurs organismes n'ont aucun plan. L'alerte auprès des autorités compétentes est transmise spontanément et de manière imprévue d'un lieu à l'autre. Plusieurs intervenants n'arrivent pas à obtenir l'information et les rapports de situation dont ils ont besoin pour fonder leurs décisions.

Les moyens de télécommunication sont insuffisants et rassemblés selon les besoins du moment. Il n'y a aucun système de radiocommunication. La transmission et la diffusion des informations sont erronées, difficiles, tardives ou dédoublées.

51 La structure de coordination adoptée ne permet pas d'avoir une perception claire et continue de l'évolution de l'événement. Elle empêche les intervenants de participer de manière efficace et immédiate aux décisions. Des directives de dernière minute contrecarrent certaines décisions dont les conséquences prévisibles ne sont pas évaluées. Des personnes ayant le pouvoir de prendre des décisions ne siègent pas sur les bons comités. Ni la fonction ni le rôle de certains intervenants majeurs ne sont connus et encore moins leur titre et leur nom.

Il n'y a pas de coordination des informations transmises au public. Ces informations sont souvent contradictoires, non pertinentes ou de l'ordre d'une opinion personnelle. Il est 
difficile de trouver un porte-parole officiel et mandaté, et de contrôler les journalistes qui s'arrachent certains intervenants, experts ou responsables politiques qui n'arrivent pas à traduire convenablement, pour le public, l'état de la situation.

Il n'y a pas de coordination entre les ministères qui donnent de l'information. Plusieurs personnes donnent des autorisations de dépenser dans le cadre d'une réglementation vague, ce qui pose des problèmes de crédits bancaires.

Le mandat des coordonnateurs désignés n'est pas très clair. Certaines personnes tardent à entrer en contact avec une autre parce qu'elle n'est pas du même ministère, du même hôpital ou du même service. Les frustrations sont nombreuses par rapport aux écarts vécus entre les attentes, les mandats et les obligations des différents intervenants. L'absence de plan crée de nombreux problèmes de partage de responsabilités.

Il est difficile de trouver une autorité morale pour coordonner les interventions d'organismes dont les responsabilités se chevauchent ou qui doivent intervenir sur des territoires contigus. Il n'y a pas de support psychosocial pour les intervenants. En plus des conflits de juridictions, bien souvent les relations personnelles l'emportent sur la mise en oeuvre de certaines ébauches de plans. Les instances locales sont écartées et les médecins coordonnateurs ne sont pas familiers avec les plans de mesure d'urgence.

\section{La vraie crise est communicationnelle}

Point n'est besoin de faire une analyse très approfondie des faits, dont il vient d'être question, pour conclure que les plans d'urgence, la communication, la télécommunication et la coordination des activités sont soumis à rude épreuve, lors d'une crise. L'origine grecque du mot crise signifie "décision». Une crise produit toujours une situation paradoxale. D'une part, il y a paralysie, incohérence et incapacité de décider et, d'autre part, urgence d'agir et de prendre les décisions pertinentes pour renouer les interactions disloquées. Les rôles habituels de certaines personnes n'ont plus de signification, alors que ceux des individus qui ont l'autorité pour agir en situation d'urgence sont exacerbés.

Les plans d'urgence sont un impératif pour retrouver les points de repères symboliques et spatio-temporels qui garantissent la certitude indispensable à la survie du tissu urbain. Toutefois, ces plans, qui sont l'énoncé d'actions d'ordre structurel et fonctionnel, tiennent pour acquis la communication et la télécommunication. Ce qui prévaut, c'est l'obsession de retrouver à tout prix et très rapidement l'état antérieur de ce qui était structuré et organisé.

Pourtant, lorsque tout s'écroule autour de nous, c'est bien la communication qui doit être prépondérante. C'est par l'intermédiaire de la communication qu'il est possible de reconstruire ou de réactiver les structures organisationnelles qui nous procurent un haut degré de certitude et de sécurité. Et la communication ne peut être efficace que si elle permet de reconstruire le monde symbolique qui s'écroule en même temps que les structures organisationnelles. Ici, la communication est beaucoup plus et autre chose que l'échange et le partage de messages. C'est d'abord un processus de création et de restructuration de réalités.

59 Les planificateurs de protocoles d'urgence auraient intérêt à comprendre que l'action d'organiser est caractérisée par le calcul, la fragmentation, la structure, la fonction, l'interaction, la certitude, l'ordre. Quant à l'action de communiquer, elle est caractérisée par la globalité, l'innovation, la transformation, la relation, l'incertitude et l'ambiguïté. Ce 
qui importe en situation de crise, c'est de reconstruire en premier lieu le système d'interrelations obligées entre l'action de communiquer et celle d'organiser, et non pas entre ce qui résulte de ces actions. C'est ce système d'interrelations qui constitue les fondements de la matrice d'actions indispensables à la reconstruction tant du monde symbolique écroulé que de la ville télé communicationnelle.

Les sociétés sont organisées autour et par l'intermédiaire de l'action de communiquer et de ses processus structurés par des expériences de relations, de production, de consommation et de pouvoir historiquement et symboliquement déterminées. En ce sens, les villes constituent les centres de communication de notre civilisation à double titre : ce sont, d'une part, des centres de création de réalités et, d'autre part, des points d'ancrage de relations humaines et d'interactions multifacettaires, multidimensionnelles et multifonctionnelles. C'est avec raison que Gottman et Harper (Gottman et Harper, 1990) considèrent que la ville est un phénomène très complexe, et même le produit artificiel le plus phénoménal et le plus sophistiqué fabriqué par l'humain. Il en est ainsi parce que l'être humain est un être de communication intrinsèquement capable de créer des réalités.

61 L'existence et la survie de ce produit artificiel, ainsi que de ceux et celles qui poursuivent sa conception et sa fabrication toujours inachevées ainsi que sa pérennité, exigent le développement et le partage d'un monde symbolique qui prend racine dans l'émergence et le maintien d'une conscience commune et non pas de rôles et de statuts. Cela s'applique non seulement aux citoyens et aux citoyennes d'une ville et à leurs élus mais aussi à ceux et celles qui ont pour mission la reconstruction du monde symbolique lors de situation d'urgence. Les massmédias ont alors une responsabilité primordiale.

62 La reconstruction du monde symbolique semble très peu préoccuper les personnes qui planifient les interventions d'urgence et, particulièrement, les journalistes qui se soucient d'abord du degré de sensationnalisme de l'événement. S'il n'existe pas un monde symbolique de substitution pour ceux et celles qui ont à intervenir en cas de sinistres ou de catastrophes - monde symbolique plus important et plus marquant que celui de la vie quotidienne et dont les significations doivent être largement partagées - comment peuton croire que ces personnes pourront agir en pleine convergence et contribuer efficacement à la restructuration de la matrice d'actions ?

Lors de sinistres ou de catastrophes, la vraie crise n'en est pas une d'organisation mais de communication. Les planificateurs de secours oublient trop souvent que c'est par l'intermédiaire de la communication qu'émergent tous les types de structures. Ils sont très peu sensibles au fait que l'être humain perçoit d'abord des relations et des systèmes de relations et non pas des objets (Watzlawick, 1967).

Chaque crise constitue donc une situation spécifique qui se traduit par des problèmes de perception, d'interaction et de représentation de la réalité. Puisque la matrice d'actions n'est plus opérationnelle, c'est la spontanéité communicationnelle qui est sollicitée. Le problème, c'est que le désir de retrouver à tout prix le degré de certitude antérieur à la situation d'urgence exige que la spontanéité communicationnelle soit organisée et structurée. Dès lors, les individus vivent une situation plutôt paradoxale

Les perceptions différentes de l'état de la situation par les répartiteurs de San Jose, les conflits de juridiction entre les intervenants et les refus de collaborer lors de l'incendie de Saint-Basile-Le-Grand, illustrent pleinement cette situation. L'effondrement du monde symbolique et des systèmes de relations qui orientaient la conduite humaine provoque 
alors le réflexe d'utiliser des systèmes de télécommunication et de diffusion, perçus d'abord comme des objets, pour retrouver au moins l'illusion d'être en interaction avec d'autres.

Les moyens de télé-communiquer peuvent être le pivot de la restructuration d'une situation chaotique essentiellement parce qu'ils permettent de fabriquer de l'espace et du temps artificiels. Par le fait même, ils sont perçus comme moyens de convergence et d'interdépendance, c'est-à-dire de restructuration des systèmes de relations perçues.

Aucun plan, aucun protocole, aucune règle ne peut remplacer le monde symbolique et relationnel perdu. Ce que ces instruments peuvent faire, c'est de transformer la conduite des intervenants en automatismes afin d'assurer un minimum de cohérence. Cette cohérence minimale durera tant que la compassion aura plus de valeur que le statut ou le rattachement institutionnel.

La convergence symbolique n'est pas fondée que sur les symboles. Les rituels, les histoires, les structures, l'esthétique et les habitants d'un quartier ou d'une ville constituent autant d'éléments de signification qui servent à créer un fond symbolique commun. L'existence de ce fond détermine à son tour, pour une grande part, le sentiment d'appartenance à partir duquel la connivence devient possible en toute situation. Si les exercices de simulation de sinistre ou de catastrophe ne peuvent créer un fond symbolique commun entre les intervenants, si ces personnes n'ont pas intégré les rituels d'intervention, si elles ne savent pas comment reconstituer la matrice d'actions et si elles ne perçoivent pas la situation de la même manière, les occasions de conflits et d'inhibition de l'action sont grandes. Par conséquent, les secours sont inefficaces, même si les systèmes de télécommunication et de diffusion continuent de fonctionner.

\section{Alléger la confusion construite}

Les villes sont devenues les centres de communication de notre civilisation. Selon Gottman et Harper (Gottmann, Harper, 1990) les technologies de télécommunication conduisent, d'une part, à la centralisation du commerce et des services dans un petit nombre de villes spécifiques dans le monde et, d'autre part, à la dispersion des activités informationnelles habituelles à la périphérie des régions métropolitaines. Les télécommunications contribuent donc à créer une nouvelle hiérarchie urbaine.

70 En situation de crise, c'est à la fois les hiérarchies urbaines, symboliques et relationnelles qu'il faut reconstruire. Or, comme les réseaux de télécommunication et les intervenants sont eux-mêmes hiérarchisés, les conflits décisionnels deviennent des parties intégrantes de toute crise.

71 Comme l'ont si bien compris Bressand et Distler (Bressand et Distler, 1985), les nouveaux réseaux informatisés de télécommunication, et l'environnement électro-photonique dont ils renouvellent constamment l'existence, font que c'est «dans l'établissement ou l'interruption de la connexion, dans l'intensité et la nature du lien aux réseaux, dans la réaction ou l'indifférence des acteurs que se détermine, à chaque instant, la physionomie du monde ». Selon ces auteurs, c'est dans ce nouveau contexte inventé par l'humain que nous sommes à la recherche du «temps hors normes » où le temps humain et le temps machine s'imbriquent l'un à l'autre permettant ainsi l'émergence du «temps à étage ».

72 La télécommunication, en tant que phénomène, transforme donc les configurations relationnelles auxquelles nous étions habitués, il y encore quelques décennies. 
Évidemment, il n'y a pas de télécommunication sans information. Celle-ci constitue à la fois la matière première et le produit des ordinateurs et des réseaux. Et ce sont les ordinateurs qui, par l'intermédiaire des algorithmes qui leur donnent vie, orchestrent de plus en plus le fonctionnement des réseaux d'interrelations communicationnelles et celui $\mathrm{du}$ tissu urbain. Les nouvelles technologies de télécommunication ont pour caractéristique fondamentale d'être beaucoup plus des processus que des produits, ce qui a pour conséquence majeure de métamorphoser les processus relationnels eux-mêmes. C'est pour cette raison que les moyens de la télécommunication envahissent si facilement tous les domaines de l'activité humaine et transforment nos manières de créer de la réalité, de produire, de consommer, de gérer, de vivre et même d'affronter une crise.

Dès lors, en situation de crise, l'espace d'intervention et les intervenants organisent leurs opérations autour des dynamiques de leur unité de production d'information. Ils interconnectent ainsi différentes fonctions à différents espaces, compte tenu des tâches assignées. Le processus global d'intervention et les actions entreprises sont donc intégrés aux manières individuelles et collectives de percevoir la télécommunication, ses technologies et l'environnement télé communicationnel.

monopolisent des segments de cet environnement télé communicationnel en s'interconnectant au-delà du quartier, de la ville, de la région et même du monde. Ce faisant, ils s'isolent des différents fragments de la société urbaine qui, en conséquence, se déstructurent dans le processus de réorganisation sélective du travail et des résidences. Les mesures de situation d'urgence tiennent peu compte de cet aspect. Pourtant, il a un effet direct sur les systèmes symboliques et relationnels à reconstruire, car il y a des choix à faire entre le local, le régional et l'international. Bhopâl, Three Mile Island, Tchernobyl et le naufrage de l'Exxon Valdez en sont quelques exemples.

75 L'analyse de divers plans d'intervention et d'études de sinistres ${ }^{1}$ fait ressortir une grande confusion entre des termes, tels que télécommunication, diffusion, communication, information. Le défaut de comprendre cela constitue une source de problèmes tant pour les planificateurs de protocoles d'intervention que pour les intervenants, dont il faudrait limiter la croissance exponentielle, lors de situation d'urgence.

76 II faudrait réserver le terme de télécommunications à tout ce qui est de l'ordre des moyens, des systèmes et des réseaux permettant aux intervenants sur les lieux d'une urgence ou d'un sinistre d'être en liaison instantanée par l'intermédiaire des ondes électromagnétiques. Les télécommunications sont indispensables pour, d'une part, constituer en système organisé les liaisons et les réseaux d'interactions vitaux et, d'autre part, pour structurer un système et un espace opérationnels de décision, de répartition, de coordination, de contrôle et de surveillance.

Le terme diffusion devrait être réservé aux moyens qui permettent de radiodiffuser ou de faire circuler des avis, des renseignements, des messages, des nouvelles ou des annonces vers les citoyens. Le terme communication devrait s'appliquer aux échanges non médiatisés entre toutes les personnes impliquées dans une situation d'urgence. Ce sont ces échanges face à face, et particulièrement les significations qui leur sont attribuées, qui font la différence entre une situation bien maîtrisée et Une situation qui évolue vers un tohu-bohu. Chaque intervenant doit savoir préalablement ce sur quoi il doit échanger, comment il doit le faire et pourquoi il doit le faire. Il doit aussi essayer de comprendre les effets possibles des messages échangés, autrement dit la signification probable et souhaitable attribuée à ces messages. 
78 Le terme information devrait faire référence, d'une part, aux renseignements transmis aux journalistes et, d'autre part, aux éléments opérationnels du contenu des messages échangés entre les différents intervenants. Cette distinction est indispensable pour que, suite à l'événement, des analyses de la situation permettent de bien départager les responsabilités et d'améliorer le contenu des divers protocoles et la performance du système d'intervention.

L'intervention, lors d'une crise, n'est pas qu'une affaire d'automatisme des gestes pertinents à poser. La reconstruction télé communicationnelle du monde symbolique et des systèmes relationnels ne peut être un automatisme fondé sur des technologies de télécommunication. Ce monde symbolique et relationnel renvoie d'abord à l'émotif et à l'affectif de chaque individu de même qu'à ses structures de perception de ce qu'il vit comme sinistré. Mais il sollicite aussi le cognitif imprégné de l'algorithmie des activités urbaines qui façonnent les perceptions et les manières de penser et d'agir, et garantit, même de façon illusoire, la certitude et l'ordre du milieu urbain.

\section{BIBLIOGRAPHIE}

BOTTERELL A., «After the Quake : Defining the Four Levels of Communications », APCO Bulletin, February 1990, pp 44-46.

BRESSAND A. et C. DISTLER, Le prochain monde, Paris, Seuil, 1985, p. 121.

GOTTMAN J. et R. A. HARPER (éds), Since Megalopolis. The Urban Writing of Jean Gottman, Baltimore, The Johns Hopkins University Press, 1990.

LEROUX R., Rapport d'évaluation de l'intervention des services de santé et des services sociaux lors de l'incendie d'un entrepôt de BPC à Saint-Basile-Le-Grand, Longueuil, Conseil de la santé et des services sociaux de la montérégie, juin 1989.

OSBORNE D. L., « Examining What Happened and Why it Happened Provides Valuable Lessons after Quake ", APCO Bulletin, February 1990, pp 9-11 et 72-73.

POWELL J. S., " The Media and Dissémination of Public Information During Emergencies », APCO Bulletin, February 1990, pp 13-15.

WATSON T., « Santa Cruz Area Hardest Hit by Quake, But Public Safety Officiais Met the Challenge », APCO Bulletin, February 1990, pp 35-37.

WATZLAWICK, Paul, Janet H. Helmick-Beavih, Don D. Jackson, Pragmatics of Human Communication, New York, Norton, 1967.

\section{NOTES}

1. Bouliane M. A., Rapport d'enquête du Coroner, Québec, Bureau du Coroner, Gouvernement du Québec, juin 1990 et novembre 1990 ; Champagne P., Plan d'un système pré hospitalier d'urgence, Québec, Conseil de la santé et des Servi ces Sociaux de la région de Québec (03), avril 1990 ; 
Leroux R., Réflexions sur le programme l'.I.A.B.S., Québec, Conseil de la santé et des Services Sociaux de la région de Québec (113), juillet 1990; Leroux, R., Rapport d'évaluation de l'intervention des services de santé et des services sociaux lors de l'incendie d'un entrepôt de BPC à Saint-Basile Legrand, Longueuil, Longueuil, Conseil de la santé et des services sociaux de la monté-régie, juin 1989 ; MSSS, Rapport du Comité aviser provincial sur le système de services pré-hospitaliers d'urgence, Québec, MSSS, version 4.0, février 1990.

\section{RÉSUMÉS}

L'auteur traite de la ville télé communicationnelle. Il rend compte de certains faits urbains dont l'existence dépend des systèmes de télécommunication. Par la suite il rapporte des faits liés à divers sinistres. Enfin, il examine différents aspects de la crise communicationnelle et télé communicationnelle lors de sinistres ou de catastrophes.

The author deals with the telecommunicationnal city. He reviews some urbain facts which existence dépends on télé communication Systems. Afterwards, he gives an account of facts about different disasters. Finally, he considers various aspects of communicational and telecommunicationnal crises at the time of disasters.

\section{AUTEUR}

\section{GILLES WILLETT}

Professeur et chercheur au Département d'information et de communication de l'Université Laval et administrateur à la Faculté des arts. Ses travaux portent sur la communication dans les organisations, le phénomène de la télécommunication et ses technologies, les théories de la communication et le droit de communiquer. Il est l'auteur de La communication modélisée, Ottawa, Les Editions du renouveau pédagogique, 1992 ainsi que De la communication à la télécommunication, Québec, Les Presses de l'Université Laval, 1989. 